\title{
Advanced Security System using RFID and IRIS Recognition System using ICA, PCA, Daugman's Rubber Sheet Model Together
}

\author{
Pankaj P. Chitte \\ persuing M.E. in Electronics \\ J.N.E.C., Aurangabad,India
}

\author{
J. G. Rana \\ HOD \& Professor in E \& TC \\ Deparment in J.N.E.C. \\ Aurangabad,India
}

\author{
Sachin Taware \\ Pravra Rural Engineering \\ College, Loni
}

\begin{abstract}
Iris recognition is the mean of biometric identification using very large amount of iris database taken without contact to the human body. Basically three main methods are available to process iris data, out of which in this paper, an iris image synthesis method based on Principal Component Analysis (PCA), Independent component analysis (ICA) and Daugman's rubber sheet model\& hybrid model is proposed. Iris Recognition is a most secure biometric authentication that uses pattern-recognition techniques. The video based iris recognition system is used to locate eye and iris, to evaluate degree of occlusion by eyelids, determine the centre \& boundary of pupil and outer edge of iris. The measured features are encoded into 512-byte iris code which is further enrolled for identification. Here we compared different techniques i.e. ICA, PCA, Daugman's rubber sheet model \& hybrid model which is combination of all above three along with RFID system. Out of 400 degrees of freedom (measurable variables), 200 features are compared to create the code which can be compared to an entire database in milliseconds. After using lot many algorithms for iris recognition we found that existing system shows, Daugman's rubber sheet model is better. The comparative study of the various algorithms proposed above shows some interesting results which is the achievement of the practical study on iris recognition.
\end{abstract}

\section{General Terms}

Iris Recognition, Daugman's rubber sheet model, Hough Transform, Histogram equalization, Gabor Filters, Gabor Wavelets etc.

\section{Keywords}

RFID, ICA, PCA, Gabber, Iris

\section{INTRODUCTION}

The different biometric authentication system available in present like fingerprint, face, and thumb are not highly secured $\&$ in that duplication is easily possible. That why to increase the security level we have planned to develop system which is combination of IRIS recognition unit and RFID unit. A variety of iris recognition approaches were proposed that can be broadly classified in three categories depending on the method used to extract features from the texture. These categories are: (1) texture-based, (2) appearance-based and (3) feature based extraction.

\subsection{Texture-based technique:}

It makes use of filters for image processing and extract iris features from the filtered images. Daugman's used Gabor wavelets to extract texture phase structure information of the iris to compute a 256-byte binary iris code. The Hamming distance measure was used to compare the difference between a pair of iris representations.

\subsection{Appearance-based technique:}

This approaches, such as Independent Component Analysis (ICA) and Principal Component Analysis (PCA), attempt to use classical statistical approaches to extract iris features. The PCA is superior in image construction, because it can control construction errors by selecting the cumulative variance. Euclidean-distance and Nearest- Neighborhood (NN) classifier are adopted in these approaches.

\subsection{Feature-based extraction techniques:}

It uses local variations, which are characterized by the appearance and disappearance of an important image structure. A bank of spatial filters is constructed for efficient feature extraction and for matching process.

For IRIS recognition we used four different types of models like ICA, PCA, Daugman's Rubber sheet model \& Hybrid model. After comparison of these algorithms we may come to know that which better one is.

\section{RFID \& IRIS RECOGNITION UNIT}

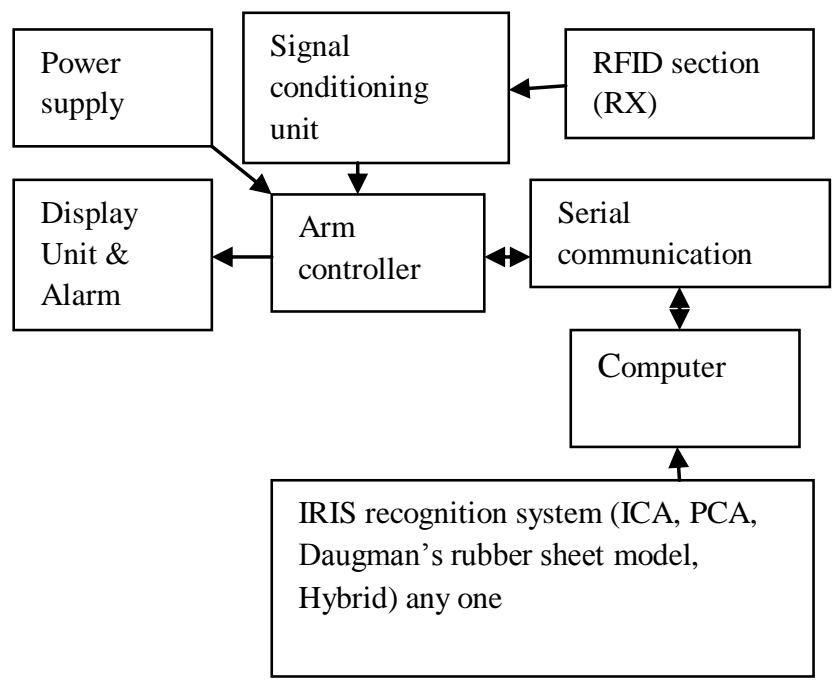

Fig.1: Iris Recognition Unit.

In this system we are going to observe two output one from RFID \& other from one of IRIS recognition system. If both outputs are high then only it will give that person is 
authenticated \& for other than these outputs it will give that person is unauthenticated which makes system more secure. These both outputs we are going to apply to ARM processor to take faster action depending on RFID \& IRIS recognition system. We are mainly refered the following paper to implement our system1) The comparison of iris recognition using principal component analysis, independent component analysis and Gabor wavelets ,Jin-Xin Shi; Xiao-Feng Gu; Computer Science and Information Technology (ICCSIT), 2010 3rd IEEE International Conference on Volume: 1 Digital Object Identifier: 10.1109/ICCSIT.2010.5563947 ,Publication Year: 2010 , Page(s): 61 - 64 [27], Mr.P.P.Chitte,Mr.J.G.Rana \& Mr.R.R.Bhambare "Iris recognition system using ICA,PCA \& Daugman's Rubersheet model together. IJCTEE,Volume 2 ,Issue 1[30], Mr.M.R.Bendre,Mr.Shivarkar S.A. An improved approach of IRIS authentication system using Daugmans rubber sheet model,segmentation, normalization and RSA security algorithm.IJCTEE,Vol.1,issue3.[21]

\subsection{Iris Authentication System}

The major goal of this system is to derive a code of iris feature scanned that would improve the recognition accuracy of an individual. It also outlines the current state of iris recognition technology. It begins with a brief overview of iris production as a means of explaining feature extraction.

\section{IRIS DETECTION PROCESS:}

\subsection{Module 1: Image Preprocessing}

\subsubsection{Graying}

In this colored image is converted in gray-pattern. Method of converting an image in gray color is by selecting two appropriate numbers that are indicated to two upper and lower thresholds (L,U). For $\mathrm{K}=1$ : iteration number do as follows:1. See the intensity of each pixel, if it is lower than the smaller $L$ $+K$, convert is to 0 and if it is bigger than $U-K$, covert it to 255. 2. Otherwise filter the intensity to the lower one by a scaling factor. The processed image is converted to a logical image that means a Black\& White type image will be obtained.
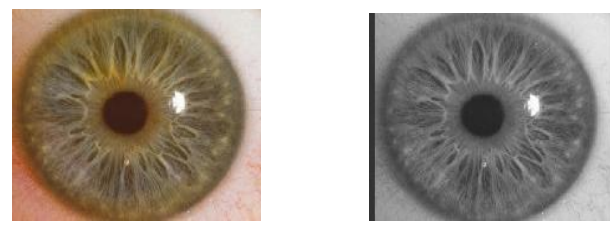

Fig.2: Original image \&Gray image.[21]

\subsection{Module 2: Segmentation}

The eyelid detection system is used to isolate most occluding eyelid regions. The iris region can be approximated by two circles, one for the iris/sclera boundary and another, interior to the first, for the iris/pupil boundary. The eyelids and eyelashes normally occlude the upper and lower parts of the iris region. Also, specular reflections can occur within the iris region corrupting the iris pattern. A technique is required to isolate and exclude these artifacts as well as locating the circular iris region.

The success of segmentation depends on the imaging quality of eye images. Persons with darkly pigmented irises will present very low contrast between the pupil and iris region if imaged under natural light, making segmentation more difficult. The segmentation stage is critical to the success of an iris recognition system, since data that is falsely represented as iris pattern data will corrupt the biometric templates generated, resulting in poor recognition rates.
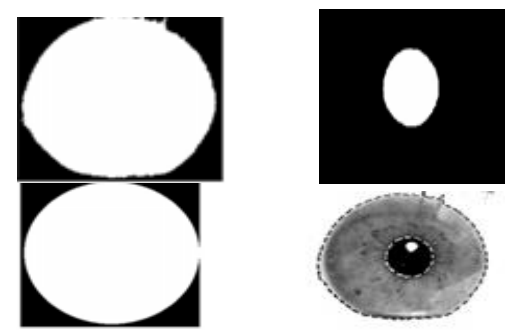

Fig.3: Inner edge $\&$ exact outer edge. \& encircled of the exact edge $\&$ the inner and outer detected boundaries.[21]

\subsubsection{Edge Detection}

For edge detection we will use Sobel operator in which gradient is calculated. The gradient is simply the derivative of the local image values. An edge in the original image would correspond to a higher value in the gradient image. Using a gradient image for the Hough transform decreases computation time significantly since only points that correspond to actual edges are used in the computation. The Sobel operator is often used to compute the gradient image as well. It has distinct advantages, although it is slightly more complex. It is less sensitive to isolated high intensity point variations because it averages points over a larger area. There are two kernels which are convolved with the image: one for each direction. The kernels are applied separately to combined to produce an absolute value[31]

\begin{tabular}{|l|l|l|}
\hline 1 & 0 & +1 \\
\hline-2 & 0 & +2 \\
\hline-1 & 0 & +1 \\
\hline
\end{tabular}

Table 1- $\mathbf{G}_{\mathbf{x}}$

\begin{tabular}{|c|c|c|}
\hline-1 & 0 & +1 \\
\hline-2 & 0 & +2 \\
\hline-1 & 0 & +1 \\
\hline
\end{tabular}

Table 2 - $\mathbf{G}_{\mathbf{y}}$
The absolute magnitude is defined as:

$$
|\mathbf{G}|=\sqrt{ }\left(\mathbf{G}_{\mathbf{x}}{ }^{2}+\mathbf{G}_{\mathbf{y}}{ }^{2}\right)
$$
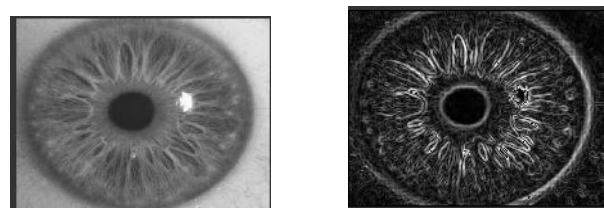

Fig.4: Original image \&Edge detected image.[21]

\subsubsection{Thresholding}

The edge and the image we have a mixture of black, gray and white values. In order to maintain the feasibility we will consider one threshold value. All the values above that threshold will be considered while and all those below as black. Thus a pure black and white image is obtained. 

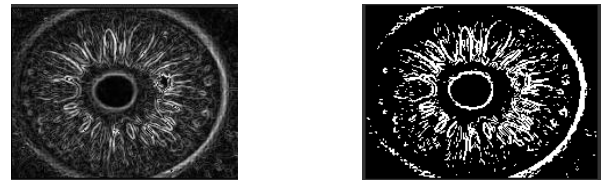

Fig.5: Edge detected image\& Threshold image.[21]

\subsubsection{Circular Hough Transform}

The transform is computed by taking the gradient of the original image (in this case, the Sobel gradient) and accumulating each non-zero point from the gradient image into every point that is one radius distance away from it. That way, edge points that lie along the outline of a circle of the given radius all contribute to the transform at the center of the circle, and so peaks in the transformed image correspond to the centers of circular features of the given size in the original image. Once a peak is detected and a circle 'found' at a particular point, nearby points (within one-half of the original radius) are excluded as possible circle centers to avoid detecting the same circular feature repeatedly.

\subsection{Module 3: Normalization}

Normalization is used to transform the iris region so that it has fixed dimensions in order to allow comparisons. The normalization process produce iris regions, which have the same constant dimensions, so that two photographs of the same iris under different conditions will have characteristic features at the same spatial location.

\subsubsection{Daugman's rubber sheet model}

The rubber sheet model remaps each point within the iris region to a pair of polar coordinates. The homogenous rubber sheet model accounts for pupil dilation, imaging distance and non-concentric pupil displacement, it does not compensate for rotational inconsistencies.

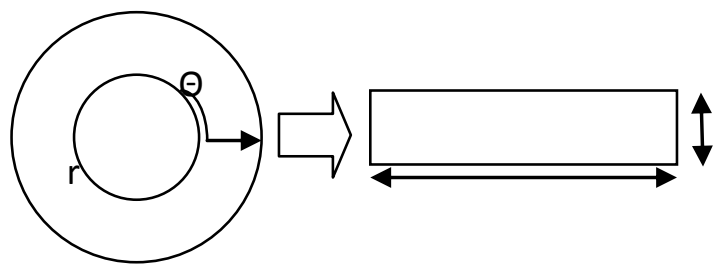

Fig.6: Daugman's rubber sheet model.[1][3][4][21][30][31]

\subsubsection{Histogram normalization}

The next stage will be to normalize the iris region in order to counteract imaging inconsistencies such as pupil dilation. An implementation of Daugman's polar representation will be used for this purpose, as this is the most documented method for iris normalization. Once a normalized iris pattern has been obtained; histogram is obtained in order to extract features.

\section{THRESHOLDING}

In this we have to consider a threshold value which is used to generate bit pattern. Individual pixels in a grayscale image are marked as "object" pixels if their value is greater than some threshold value (assuming an object to be brighter than the background) and as "background" pixels otherwise. Typically, an object pixel is given a value of " 1 " while a background pixel is given a value of " 0 ."

\subsubsection{Bit Pattern Generation}

Since an individual iris region contains features with high degrees of freedom, each iris region will produce a bit-pattern which is independent to that produced by another iris, on the other hand, two iris codes produced from the same iris will be highly correlated.

\subsection{Module 4: Pattern Matching}

For pattern matching we have used the same concept that is used in OCR for character recognition i.e we will match the two bits if match is found some score value is added and that goes on increasing accordingly and if not found we start with the negative value of the starting score value. On the basis of threshold score decision of valid iris is made and authentication is done.

\subsection{Module 5: Encryption / Decryption Of Data}

The RSA algorithm can be used for public key encryption. The 3 basic steps of this algorithm are: 1.Key Generation Algorithm 2.Encryption 3.Decryption

\subsubsection{Key Generation Algorithm}

1. Generate two large random primes, p and q, of approximately equal size such that their product $n=$ $\mathrm{pq}$ is of the required bit length, e.g. 1024 bits.

2. Compute $\mathrm{n}=\mathrm{pq}$ and $(\varphi) \mathrm{phi}=(\mathrm{p}-1)(\mathrm{q}-1)$.

3. Choose an integer e, $1<\mathrm{e}<$ phi, such that $\operatorname{gcd}(\mathrm{e}$, phi) $=1$.

4. Compute the secret exponent $\mathrm{d}, 1<\mathrm{d}<$ phi, such that ed $=1$ (mod phi).

5. The public key is (n, e) and the private key is (n, d). The values of $p, q$, and phi should also be kept secret.

- $\mathrm{n}$ is known as the modulus.

- $\quad \mathrm{e}$ is known as the public exponent or encryption exponent.

- $\mathrm{d}$ is known as the secret exponent or decryption exponent.

\subsubsection{Encryption}

Sender A does the following:-

1. Obtains the recipient B's public key $(n, e)$.

2. Represents the plaintext message as a positive integer $\mathrm{m}$.

3. Computes the cipher text $\mathrm{c}=\mathrm{m}^{\wedge \mathrm{e}} \bmod \mathrm{n}$.

4. Sends the cipher text $\mathrm{c}$ to $\mathrm{B}$.

\subsubsection{Decryption}

Recipient B does the following:-

Uses his private key $(n, d)$ to compute,

$\mathbf{m}=\mathbf{c}^{\wedge} \mathbf{d} \bmod \mathbf{n}$.

Extracts the plaintext from the integer representative $\mathrm{m}$.

\subsubsection{Iris Recognition Algorithm Based on PCA}

An iris recognition based on PCA is first presented to put iris synthesis in context. Most existing iris recognition methods are based on the local properties. as phase, shape, and so on. However, iris image synthesis

based on local properties is difficult to implement. Recently, Bae et al. attempted to use the Independent Component Analysis (ICA) to extract iris feature. and PCA can all be used for global feature extraction, PCA has superiority in image construction, because we can control the construction 
errors by selecting the cumulative variance. Euclidean distance and nearest neighborhood (NN) classifier are adopted here.

When should PCA be used?

- In community ecology, PCA is useful for summarizing variables whose relationships are approximately linear or at least monotonic e.g. A PCA of many soil properties might be used to extract a few components that summarize main dimensions of soil variation

- $\quad$ PCA is generally NOT useful for ordinating community data

- Why? Because relationships among species are highly nonlinear.

Algorithm

Problem Definition: The iris is used to authenticate a person.

The objectives are:

i) Reducing the complexity of the algorithm by eliminating edge detection and normalization.

ii) To improve accuracy of recognition The algorithm of PIRDWT to recognize a person using iris localization DWT, PCA and multiclassifiers is given in

\section{Algorithm of PIRDWT:}

Input: Eye image

Output: Recognition of a person

Step 1: Read the eye image.

Step 2: Iris template creation.

Step 3: Histogram equalization on the iris template.

Step 4: The coiflet wavelet is applied and the approximation band is considered.

Step 5: PCA is applied on approximation band to form feature vector.

Step 7: Form the signature of each image.

Step 8: Perform steps 1 to 7 for test image.

Step 9: Match/Non match decision is obtained using multiclassifiers.

\section{Flowchart: $[21][22]$}

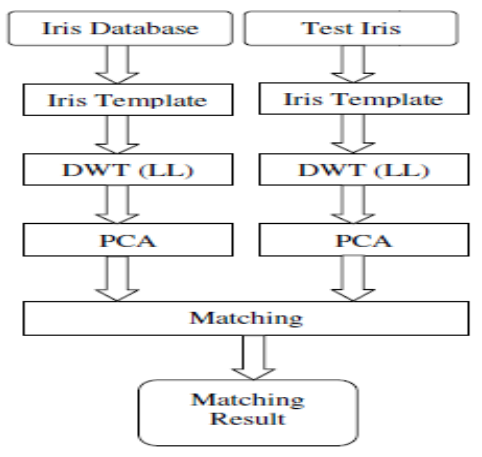

\section{ICA Algorithm}

Fig. 6:

\section{a. Introduction}

When the independence assumption is correct, blind ICA separation of a mixed signal gives very good results. It is also used for signals that are not supposed to be generated by a mixing for analysis purposes. A simple application of ICA is the "cocktail party problem", where the underlying speech signals are separated from a sample data consisting of people talking simultaneously in a room. Usually the problem is simplified by assuming no time delays or echoes. An important note to consider is that if $N$ sources are present, at least $N$ observations (e.g. microphones) are needed to get the original signals. This constitutes the square $(J=D$, where $D$ is the input dimension of the data and $J$ is the dimension of the model). Other cases of underdetermined $(J<D)$ and over determined $(J>D)$ have been investigated.

\section{b. Defining Component Independence}

ICA finds the independent components (aka factors, latent variables or sources) by maximizing the statistical independence of the estimated components. We may choose one of many ways to define independence, and this choice governs the form of the ICA algorithms. The two broadest definitions of independence for ICA are

1) Minimization of Mutual Information

2) Maximization of non-Gaussianity

The Non-Gaussianity family of ICA algorithms, motivated by the central limit theorem, uses kurtosis and negentropy. The Minimization-of-Mutual information (MMI) family of ICA algorithms uses measures like Kullback-Leibler Divergence and maximum-entropy. The typical algorithms for ICA use centering, whitening (usually with the eigen value decomposition), and dimensionality reduction as preprocessing steps in order to simplify and reduce the complexity of the problem for the actual iterative algorithm. Whitening and dimension reduction can be achieved with principal component analysis or singular value decomposition. Whitening ensures that all dimensions are treated equally a priori before the algorithm is run. Algorithms for ICA include infomax, Fast ICA, and JADE, but there are many others also.

In general, ICA cannot identify the actual number of source signals, a uniquely correct ordering of the source signals, nor the proper scaling (including sign) of the source signals.

ICA is important to blind signal separation and has many practical applications. It is closely related to (or even a special case of) the search for a factorial code of the data, i.e., a new vector-valued representation of each data vector such that it gets uniquely encoded by the resulting code vector (loss-free coding), but the code components are statistically independent.

\section{c. Mathematical definitions:}

Linear independent component analysis can be divided into noiseless and noisy cases, where noiseless ICA is a special case of noisy ICA. Nonlinear ICA should be considered as a separate case.

\section{d. General definition:}

The data is represented by the random vector $\mathbf{x}=\left(\mathbf{x}_{1}, \ldots \ldots \ldots \mathbf{x}_{\mathbf{m}}\right)$ and the components as the random vector $\mathbf{s}=\left(\mathbf{s}_{1}, \ldots \ldots \ldots . \mathbf{s}_{\mathbf{m}}\right)$. The task is to transform the observed data $\mathbf{x}$, using a linear static transformation $W$ as

$\mathbf{S}=\mathbf{W}_{\mathbf{x}}$,

into maximally independent components $S$ measured by some function $\mathbf{F}\left(\mathbf{s}_{1}, \ldots \ldots \ldots \mathbf{s}_{\mathbf{n}}\right)$ of independence.

\section{DAUGMANS RUBBER SHEET MODEL (GABOR FILTOR)}

The homogenous rubber sheet model devised by Daugman [1] remaps each point within the iris region to a pair of polar coordinates $(r, \grave{e})$ where $r$ is on the interval $[0,1]$ and $\grave{e}$ is angle $[0,2 \pi]$. 


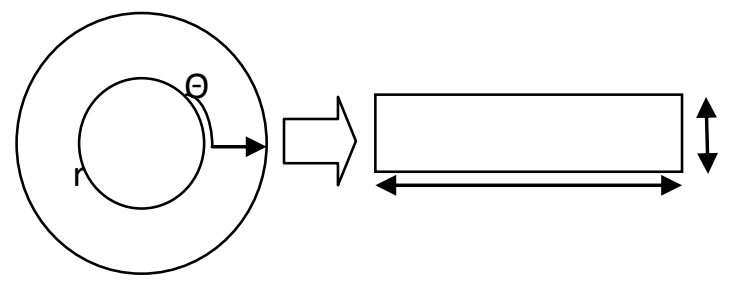

Fig. 7: Daugman's rubber sheet model.

The remapping of the iris region from $(x, y)$ Cartesian coordinates to the normalized non-concentric polar representation is modeled as

$\mathrm{I}(\mathrm{x}(\mathrm{r}, \boldsymbol{\theta}), \mathrm{y}(\mathrm{r}, \boldsymbol{\theta}))) \longrightarrow \mathrm{I}(\mathrm{r}, \boldsymbol{\theta}))$

With

$\mathbf{x}(\mathbf{r}, \theta))=(1-\mathbf{r}) \mathbf{x}_{\mathrm{p}}(\theta)+\mathbf{r x}_{1}(\theta)$

$y(r, \theta))=(1-r) y_{p}(\theta)+r y_{1}(\theta)$

Where, $I(x, y)$ is the iris region image, $(x, y)$ are the original Cartesian coordinates, $(r, \grave{e})$ are the corresponding normalized polar coordinates, and are the coordinates of the pupil and iris boundaries along the $\grave{e}$ direction. The rubber sheet model takes into account pupil dilation and size inconsistencies in order to produce a normalized representation with constant dimensions. In this way the iris region is modeled as a flexible rubber sheet anchored at the iris boundary with the pupil centre as the reference point.

Even though the homogenous rubber sheet model accounts for pupil dilation, imaging distance and non-concentric pupil displacement, it does not compensate for rotational inconsistencies. In the Daugman system, rotation is accounted for during matching by shifting the iris templates in the $\grave{e}$ direction until two iris templates are aligned.

\section{WAVELET ENCODING}

Wavelets can be used to decompose the data in the iris region into components that appear at different resolutions. Wavelets have the advantage over traditional Fourier transform in that the frequency data is localized, allowing features which occur at the same position and resolution to be matched up. A number of wavelet filters, also called a bank of wavelets, is applied to the 2D iris region, one for each resolution with each wavelet a scaled version of some basis function. The output of applying the wavelets is then encoded in order to provide a compact and discriminating representation of the iris pattern.

\section{GABOR FILTERS}

Gabor filters are able to provide optimum conjoint representation of a signal in space and spatial frequency. A Gabor filter is constructed by modulating a sine/cosine wave with a Gaussian. This is able to provide the optimum conjoint localization in both space and frequency, since a sine wave is perfectly localized in frequency, but not localized in space. Modulation of the sine with a Gaussian provides localization in space, though with loss of localization in frequency. Decomposition of a signal is accomplished using a quadrature pair of Gabor filters, with a real part specified by a cosine modulated by a Gaussian, and an imaginary part specified by a sine modulated by a Gaussian. The real and imaginary filters are also known as the even symmetric and odd symmetric components respectively. [21][31]

\section{RESULTS}

1. Create GUI

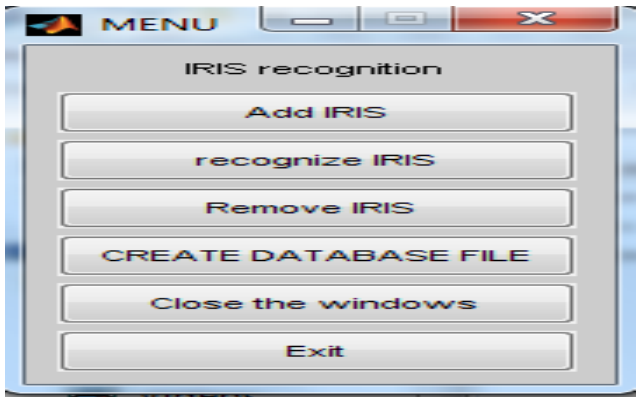

\section{Add IRISImage}

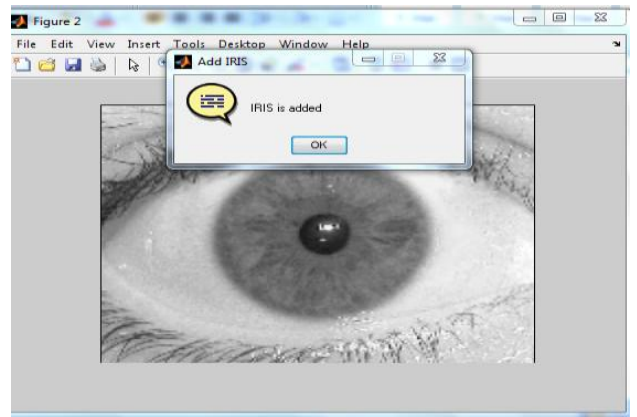

3. Image Match

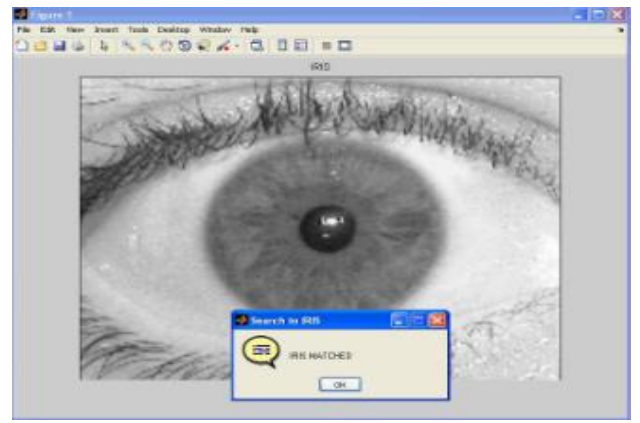

Fig 8: Graph of distance between input error \& validation error in ICA

Table 3: Distance between input error \& validation error

\begin{tabular}{|l|l|l|l|}
\hline ICA & PCA & DAUGMAN & $\begin{array}{l}\text { HYBRI } \\
\text { D }\end{array}$ \\
\hline $2.18 \mathrm{E}-10$ & $3.26 \mathrm{E}-10$ & $8.26 \mathrm{E}-07$ & $9.69 \mathrm{E}-06$ \\
\hline $5.14 \mathrm{E}-07$ & $5.14 \mathrm{E}-07$ & $3.11 \mathrm{E}-07$ & $8.93 \mathrm{E}-06$ \\
& & & \\
\hline $2.09 \mathrm{E}-10$ & $2.09 \mathrm{E}-10$ & $2.79 \mathrm{E}-10$ & $9.02 \mathrm{E}-06$ \\
& & & \\
\hline
\end{tabular}




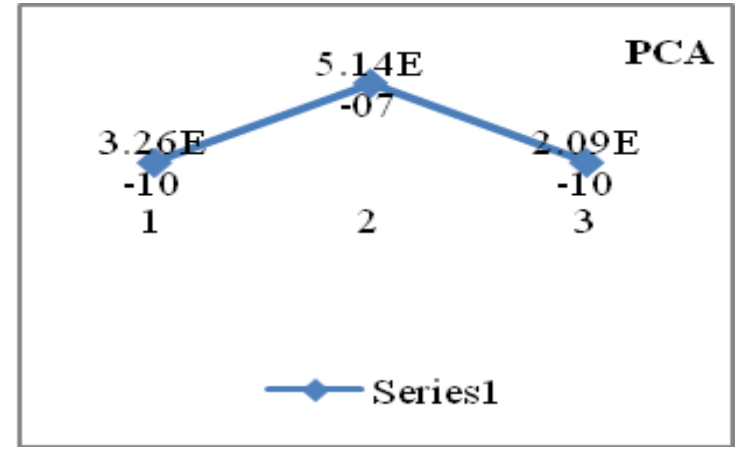

Fig 9: Graph of distance between input error \& validation error in PCA

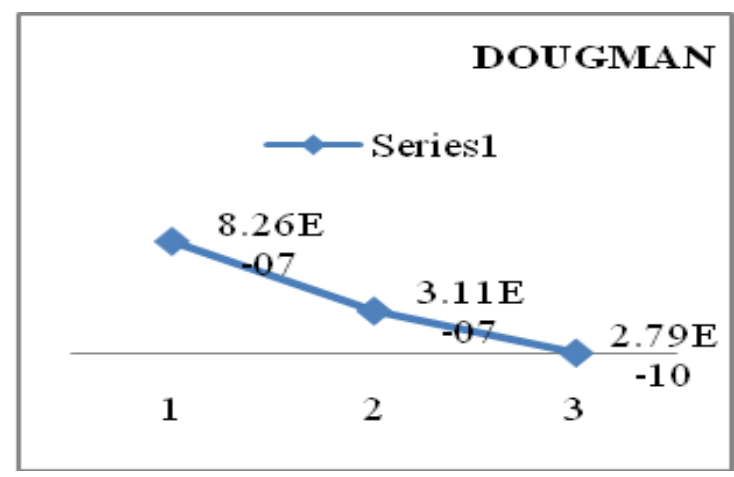

Fig 10: Graph of distance between input error \& validation error in Daugman

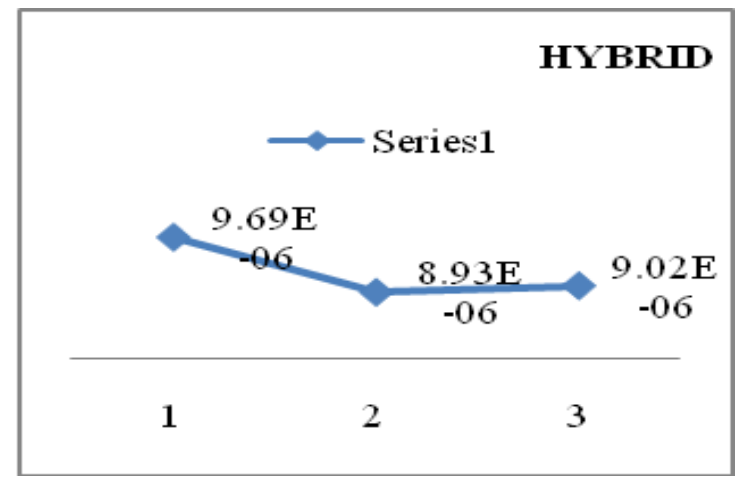

Fig 11: Graph of distance between input error \& validation error in Hybrid

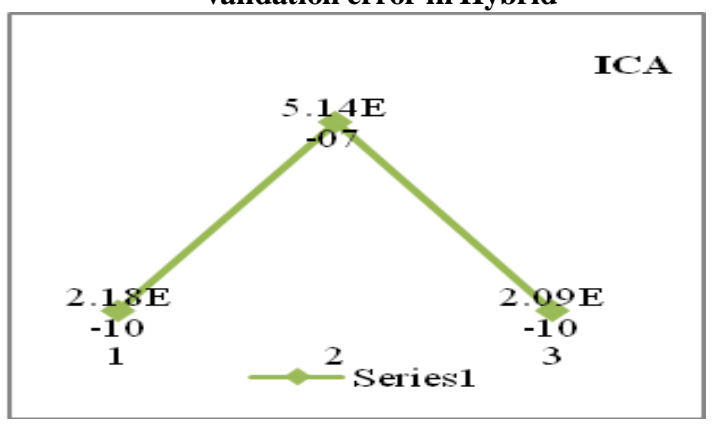

In this we have taken reading of three diffrent images for each method(ICA,PCA,Daugman \& Hybrid).To measure performance we have calculated distance between input error $\&$ validation error for each image.And if distance between input error \& validation error for image is small then performance is good \& performance is poor for large distance.As we observe readings \& graphs its clear that sequnce of performance(good to less good) is ICA,PCA are nearly same,thenhybrid and Dugmans.

\section{CONCLUSION}

In this paper, iris recognition algorithms are compared and also we have increased the security using RFID. The need for secure methods of authentication is becoming increasingly important. Currently, highly accurate personal recognition is feasible using the human iris mainly because of its stability throughout a lifetime and its uniqueness. Iris recognition systems are relatively compact and efficient and have shown promising performance. In our paper we have successfully implemented the IRIS Authenticated System with RFID to make more secure system \& removing its previous drawback and improvising it. We also have extended our project by using combination of RFID \& hybrid IRIS recognition. Thus towards conclusion we can say that

- Iris authentication is the most feasible because of use of three algorithm together (i.e. ICA, PCA \& Daugman's Rubber Sheet Model) \& RFID which shows compact and efficient and have promising performance.

- We are going to take images from UBIRIS, a standard database in which there are 230 sets of images with each set having 5 images of a single person and we think that after testing we will get more efficient result than existing system.

- Last most important thing which can be observed from results is that after reading we got the performance of ICA is better compare to other system according to our implementation which is complete exception to the consideration of superiority of Gabber's method.

\section{REFERENCES}

[1] J. Daugman. How iris recognition works. Proceedings of 2002 International Conference on Image Processing, Vol. $1,2002$.

[2] R. Wildes. Iris recognition: an emerging biometric technology. Proceedings of the IEEE, Vol. 85, No. 9, 1997.

[3] J. Daugman. Biometric personal identification system based on iris analysis. United States Patent, Patent Number: 5,291,560, 1994

[4] J. Daugman. High confidence visual recognition of persons by a test of statistical independence. IEEE Transactions on Pattern Analysis and Machine Intelligence, Vol. 15, No. 11, 1993.

[5] L. Masek,"Recognition of Human Iris Patterns for Biometric Identification", M. Thesis. The University Of Western Australia, 2003.

[6] HI W. W. Boles and B. Boashash. "A human identification technique using images of the iris and wavelet transform', IEEE Transactions on Signal Processing, vol. 46, no. 4, pp. 1185-1188, 1998.

[7] J. G. Daugman, "Complete discrete 2-D Gabor transforms by neural networks for image analysis and compression", IEEE Trans. Acoust., Speech, Signal Processing, vol. 36, pp. 11691179, 1988.

[8] D. Gabor, "Theory of communication", J. Inst. Elect. Eng., vol. 93, pp. 429459, 1946. 
[9] J. Huang, L. Ma, Y. Wang, and T. Tan, "Iris recognition based on local orientation description", in Proc. 6th Asian Conf. Computer Vision, vol. II, 2004, pp. 954959.

[10] A. K. Jain, R. M. Bolle, and S. Pankanti, Eds., Biometrics: Personal Identification in Networked Society, Norwell. MA: Kluwer, 1999. [21 D. Zhang, AutomatedBiometrics: Technologies andSystems . Norwell, MA: Kluwer, May 2000.

[11] L. Ma, Y. Wang, and T. Tan, "Iris recognition using circular symmetric filters", in Proceedings of the 16th International Conference on Pattern Recognition, vol. 2, pp. 414417, 2002.

[12] L.Ma, T. Tan, Y.Wang, and D. Zhang, "Personal identification based on iris texture analysis", IEEE Transactions on Pattern Analysis and Machine Intelligence, vol. 25, no. 12, pp. 1519 1533,2003. [101 L. Ma, T. Tan,D. Zhang, andYWang, "Local intensity variation analysis for iris recognition", Pattern Recognition, vol. 37, no. 6, pp. 12871298, 2005. [Ill L- Ma, Y. Wang, and T. Tan, "Iris recognition based on multichannel Gabor filtering", in Proc. 5th Asian Conf. Computer Vision, vol. I, 2002, pp. 279283. [121 C. Park, J. Lee, M. Smith, and K. Park, "Iris-based personal authentication using a normalized directional energy feature", in Proc. 4th Int. Conf. Audio- and Video-Based Biometric Person Authentication, 2003, pp. 224232.

[13] H. Proenca and L. A. Alexandre, "UBIRIS: A noisy iris image database", Proc. 13th International Conference on Image Analysis and Processing (1CJAP2005), pp. 970977 ,

[14] H. Proenca and L. A. Alexandre, "Iris segmentation methodology for non-cooperative iris recognition", IEE Proc. Vision, Image \& Signal Processing, vol. 153, issue 2, pp. 199-205, 2006.

[15] R- Sanchez-Reillo and C. Sanchez-Avila, "Iris recognition with low template size", in Proc. Int. Conf. Audio- and Video-Based Biometric Person Authentication, 2001, pp. 324329.

[16] Z. Sun, Y. Wang, T. Tan, J. Cui, " Improving iris recognition accuracy via cascaded classifiers", IEEE Trans, on Systems, Man, and Cybernetics-Part C", vol.35, no.3, pp.435-441,2005.

[17] C. Tisse, L. Martin, L. Torres, and M. Robert, "Person identification technique using human iris recognition", in Proc. Vision Interface, 2002, pp. 294299.

[18] R. R Wildes, "Iris recognition: an emerging biometric technology", Proceedings of the IEEE, vol. 85, no.9, pp. 1348-1363, 1997.

[19] R- P. Wildes, J. C. Asmuth, G. L. Green, S. C. Hsu, R. J. Kol-czynski, J. R. Matey, and S. E. McBride, "A machine vision system for iris recognition", Much. Vision Applicat., vol. 9, pp. 18, 1996.

[20] X. Yuan and P. Shi, "A non-linear normalization model for iris recognition". Proceedings of the International
Workshop on Biometric Recognition Systems IWBRS 2005, pp. 135-142, China, 2005.

[21] Mr.M.R.Bendre,Mr.Shivarkar S.A. An improved approach of IRIS authentication system using Daugmans rubber sheet model,segmentation, ,normalization and RSA security algorithm.IJCTEE,Vol.1,issue3.

[22] PCA based Iris recognition using DWT by Shashikumar D.R.,K.B.Raja,R.K.Chhoottaray,Sabyasachi Pattnaik in et,al,Int. Journal comp. tech.Appl.,Vol 2(4),884- 893.

[23] Face recognition using ICA by Marian Stewart Bartlett,Javier Movellen,Terrence Sejnowski in IEEE Transaction on neural networks Vol.13,No.6 November 2002

[24] Independent Component Analysis: Theory and Applications,

[25] Te-Won Lee, Kluwer Academic Publishers, September 1998,ISBN: 0792382617

[26] Improving feature vectors for iris recognition through design and implementation of new filter bank and locally compound using of PCA and ICA .Ranjzad, H.; Ebrahimi, A.; Sadigh, H.E.; Applied Sciences on Biomedical and Communication Technologies, 2008. ISABEL '08. First International Symposium on Digital Object Identifier: 10.1109/ISABEL.2008.4712612 ,Publication Year: 2008 , Page(s): 1 - 5

[27] The comparison of iris recognition using principal component analysis, independent component analysis and Gabor wavelets ,Jin-Xin Shi; Xiao-Feng Gu; Computer Science and Information Technology (ICCSIT), 2010 3rd IEEE International Conference on Volume: 1 Digital Object Identifier: 10.1109/ICCSIT.2010.5563947 ,Publication Year: 2010 , Page(s): 61 - 64

[28] Feature Extraction of Iris Images using ICA for Person Authentication ,Talbar, S.N.; Bodade, R.M.; Signal Processing and Communications, 2007. ICSPC 2007. IEEE International Conference on Digital Object Identifier: 10.1109/ICSPC.2007.4728504 ,Publication Year: 2007 , Page(s): 1055 - 1058

[29] Performance evaluation of Independent Component Analysis in an iris recognition system ,Bouraoui, I.; Chitroub, S.; Bouridane, A.; Computer Systems and Applications (AICCSA), 2010 IEEE/ACS International Conference on Digital Object Identifier: 10.1109/AICCSA.2010.5586977 Publication Year: 2010 , Page(s): 1 - 7

[30] Mr.P.P.Chitte,Mr.J.G.Rana \& Mr.R.R.Bhambare "Iris recognition system using ICA,PCA \& Daugman's Rubersheet model together. IJCTEE,Volume 2 ,Issue 1

[31] Mahboubeh Shamsi, Abdolreza Rasouli "A Novel Approach for Iris Segmentation and Normalization" Faculty of Computer Science \& Information System Islamic Azad University. 